\title{
Standing wave description of nearly conservative, parametrically driven waves in extended systems
}

\author{
Francisco J. Mancebo, José M. Vega* \\ E.T.S.I. Aeronánaticos. P/. Cardenal Cisneros 3. Universidad Politécnica de Madrid, 28040 Madrid. Spain
}

\begin{abstract}
We consider the standing wavetrains that appear near threshold in a nearly conservative. parametrically excited, extended system that is invariant under space translations and reflection. Sufficiently close to threshold, the relevant equation is a GinzburgI,andau equation whose cubic coefficient is extremely sensitive to wavenumber shifts. which can only be understood in the context of a more general quintic equation that also includes two cubic terms involving the spatial derivative. This latter equation is derived from the standard system of amplitude equations for counterpropagating waves, whose validity is well established today. The coefficients of the amplitude equation for standing waves are obtained for ID Faraday waves in a decp container, to correct several gaps in former analyses in the literature. This application requires to also consider the effect of the viscous mean flow produced by the surface waves, which couples the dynamics of the surface waves themselves with the free surface deformation induced by the mean flow.
\end{abstract}

PACS: 47.20.Ky: 47.20.Ma; 47.35.+i; 47.54.+r

Keywords: Nearly conservative systems; Amplitude equations; Parametrically driven waves: Standing Faraday waves; Mean flow

\section{Introduction}

This paper deals with the weakly nonlinear dynamics of parametrically driven wavetrains in a nearly conservative, extended system that is invariant under the orthogonal group $O(2)$ generated by space translations and reflection. These waves exhibit a frequency that is a half of the forcing frequency and a nonzero wavenumber, and appear as the forcing amplitude exceeds a threshold value. The simplest case is that in which the system is large only in one direction. Because of invariance under reflection, the waves either propagate to the left or to the right. 
But, at sufficiently small amplitude (namely, sufficiently close to threshold) the parametric forcing favors an equal superposition of these waves that builds a standing wave, hereafter SW. A first aim of this paper is to derive and discuss the amplitude equation for the evolution of this $S W$. In addition, we shall consider the Faraday system at small viscosity, which is by far the most studied example of nearly conservative, parametrically forced, extended system. The waves named after Faraday [1] are gravily-capillary surface waves that are parametrically excited by vertical vibration of the container $[2-4]$. Unfortunately, this is not the simplest example to illustrate the analysis below. This is because the correct description of the Faraday system requires to consider a viscous mean fow, as already pointed out by one of us in a series of papers [5-8]. But even ignoring the mean flow, as we shall do for the sake of clarity in the remaining of this section, there is no a systematic derivation in the literature of the amplitude equations that directly describe the SWs near threshold in the small viscosity limit, and current approaches exhibit some essential deficiencies, whose correction is the main object of this paper.

In fact, these deficiencies arose when we took the limit of small viscosity in the results of our weakly nonlinear analysis of viscous Faraday waves near threshold [9]. In particular, the coefficient of the cubic tem in the SW-amplitude equation showed a significant discrepancy when compared with current nearly inviscid calculations in the literature (see Fig. 2c below), which in turn had been already controversial [10]. When analyzing the discrepancy, which was not due to any mistake in the calculations in [9] (as checked with former independent results by Chen and Viñals [11], see Fig. 2c below) we encountered a chain of mistakes in current analy ses at small viscosity $(\varepsilon \ll 1)$. Namely:

A. We checked that the cubic term, $\beta_{5}(\sim 1)$, in the SW-amplitude equation (namely, Eq. (3) below) is extremely sensitive to the wavenumber $k$ : it shows a $O(1)$-correction when $k$ is varied by a $O\left(\varepsilon^{2}\right)$ quantity. The wavenumber at threshold (centered at its inviscid approximation) is of the form [11] (see Eq. (51) below)

$$
k_{\ell}=k_{1} \varepsilon^{3 / 2}+k_{2} \varepsilon^{2}+\cdots \text {. }
$$

This yields a first mistake because current calculations are made at $k_{c}=0$. But this is a subtle matter because $k_{c}$ can be decomposed into two parts, $k_{c}=k_{c 0}+\kappa_{c}$, where $k_{c: 0}=k_{1} \varepsilon^{3 / 2}+k_{20} \varepsilon^{2}+\cdots$ is produced by viscous effects only and can be climinated from the outset by a change of variable, see below; thus the shift $k_{t: 0}$ has no effect in $\beta_{5} . \kappa_{c}=k_{21} \varepsilon^{2}+\cdots$ instead is due to parametric forcing and has a $O(1)$ effect on $\beta_{5}$ that can be calculated analytically. This effect can be added to current results in the literature (which ignored it), but unfortunately a (smaller but still) $O(1)$ discrepancy remains. Therefore, additional gaps must be present in existing (asymptotic) computations of $\beta_{5}$ and the whole asymptotic calculation of $\beta_{5}$ must be carefully repeated. But this (quite tedious) asymptotic analysis is beyond the scope of this paper. The asymptotic value of $\beta_{5}$ is calculated below letting $\varepsilon \rightarrow 0$ in the viscous calculations in [9].

B. The extreme sensitivity of $\beta_{5}$ to the wavenumber shift is not compatible with the SW-amplitude equation guessed by Milner [12], which has been uncritically taken for granted in the literature. When conveniently scaled (see Section 3.1 below), this equation is

$$
A_{t}=\frac{\beta_{1}}{\varepsilon} A_{x x}+\beta_{0}\left(\mu-\mu_{c}\right) A-\frac{\beta_{2}}{\varepsilon}|A|^{4} A-\varepsilon \beta_{5}|A|^{2} A,
$$

where $\mu_{c} \sim \varepsilon$ is the threshold value of the forcing amplitude $\mu$ and $\beta_{0} \sim \beta_{1} \sim \beta_{2} \sim \beta_{5} \sim 1$. Eq. (2) should apply in a close neighborhood of threshold $\left(\left|k-k_{c}\right| \sim \varepsilon^{2},\left|\mu-\mu_{c}\right| \sim \varepsilon^{3}\right.$, see Fig. 1) and reduces to the standard Ginzburg-Landau (GL) equation with real coefficients,

$$
A_{t}=\frac{\beta_{1}}{\varepsilon} A_{x x}+\beta_{0}\left(\mu-\mu_{c}\right) A-\varepsilon \beta_{5}|A|^{2} A,
$$

in a still closer $\left(\left|k-k_{c}\right| \ll \varepsilon^{2},\left|\mu-\mu_{c}\right| \ll \varepsilon^{3}\right)$ neighborhood (Fig. 1). Up to rescaling, this is the usual equation that describes the weakly nonlinear response near threshold of parametrically driven waves in fully dissipative systems [9]. Now, a $O\left(\varepsilon^{2}\right)$-wavenumber shift is accounted for replacing $A$ by $\Lambda \mathrm{e}^{\mathrm{i} \varepsilon^{2} \delta x}$, which adds (new terms 


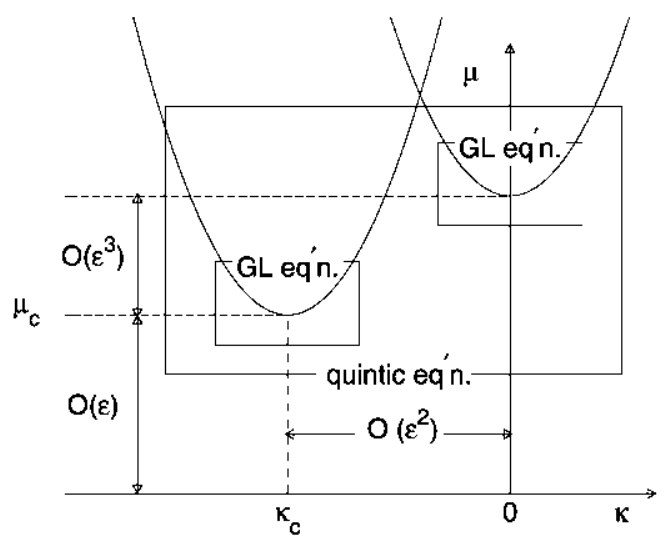

Fig. 1. The neutral instability curves of the spatially uniform SWs of (21) ignoring the effect of the wavenumber shift (curve on the right) and laking this effect into account (curve on the left). Note that the wavenumber shilt is too large to be appreciated in that part of the marginal instability curves associated with the Gl. Eq. (3), but is in the scope of the quintic Eq. (4). Milner's quintic Eq. (2) would also apply in the larger domain. $|\kappa| \sim \varepsilon^{2},|\mu-| \mu_{c} \mid \sim \varepsilon^{3}$.

proportional to $i \varepsilon A_{x}$ and $\varepsilon^{3} A$ but) no correction to $\beta_{5}$. The $O(1)$-effect on $\beta_{5}$ requires the presence of at least one new term, namely a term proportional to $\mathrm{i} \varepsilon^{-1}|A|^{2} A_{x}$. The correct equation is

$$
A_{i}=\frac{\beta_{1}}{\varepsilon} A_{x x}+\beta_{0}\left(\mu-\mu_{c}\right) A-\frac{\beta_{2}}{\varepsilon}|A|^{4} A-\mathrm{i} \frac{\beta_{3}}{\varepsilon}\left(|A|^{2}\right)_{x} A-\mathrm{i} \frac{\beta_{4}}{\varepsilon}|A|^{2} A_{x}-\varepsilon \beta_{5}\left|A^{2}\right| A .
$$

The new terms in (4) ( $\mathrm{cf}(2)$ are of the same order as the remaining nonlinear terms in the distinguished limit

$$
|A|^{2} \sim\left|\frac{\partial}{\partial x}\right| \sim \varepsilon^{2}, \quad\left|\mu-\mu_{c:}\right| \sim\left|\frac{\partial}{\partial t}\right| \sim \varepsilon^{3},
$$

which is defined such that all terms in Eq. (4) are comparable. As $\left|\mu-\mu_{c}\right| \ll \varepsilon^{3}$ we obtain (after appropriate rescaling) the GL Eq. (3) again. For larger $\left|\mu-\mu_{c}\right|$, as $\varepsilon^{3} \ll\left|\mu-\mu_{c}\right| \ll \varepsilon$, Eq. (4) can be rescaled such that all terms except the cubic one (which becomes smaller) be still comparable, namely Eq. (4) can be replaced by

$$
A_{i}=\frac{\beta_{1}}{\varepsilon} A_{x x}+\beta_{0}\left(\mu-\mu_{c}\right) A-\frac{\beta_{2}}{\varepsilon}|A|^{4} A-\mathrm{i} \frac{\beta_{3}}{\varepsilon}\left(|A|^{2}\right)_{x} A-\mathrm{i} \frac{\beta_{4}}{\varepsilon}|A|^{2} A_{x} .
$$

The upper restriction for the scope of $(6),\left|\mu-\mu_{c}\right| \ll \varepsilon$, results from the fact that as $\left|\mu-\mu_{c}\right| \sim \varepsilon$ or larger, the description in terms of SWs is not the relevant one and the two counterpropagating waves that build the SW must be considered separately, see $[5,8]$.

The SW-amplitude equations (4) and (6) are new, to our knowledge. They both exhibit two cubic terms involving the spatial derivatives, which (a) break a spurious reflection symmetry because both (4) and (6) are invariant under the action

$$
(x, A) \rightarrow(-x, \bar{A}) \text {. }
$$

which results from the reflection symmetry of the original problem, while both (2) and (3) are invariant under the actions $x \rightarrow-x$ and $A \rightarrow A$ separately; and (b) prevent the existence of a Lyapunov function, which exists for both (2) and (3), as is readily seen. Properties (a) and (b) are expected to have a dramatic effect in the dynamics and, in particular, to allow non steady attractors.

Although the more interesting consequences of the corrections to previous theories mentioned above manifest themselves in two-dimensional Faraday waves (which require a 3D container), in order to clearly explain the nature 
of the difficulty that led to wrong results, we shall consider the simplest one-dimensional case. And since the same difficulty appears in other nearly conservative, extended systems that do not produce any mean flow, we shall first treat the problem at this level of generality. The difficulty can be seen (and the correct SW-equation can be guessed) in a quite simple (but careful) analysis, which will be performed first, in Section 2. But a complete derivation of the SW-equation is necessary and will be presented in Section 3 in the distinguished limit (5). Comparison with exact results for the Faraday system will be made in Section 4, where the effect of the mean flow will also be added. The paper ends with some concluding remarks on the scope, consequences, and extensions of the results below.

\section{A preliminary analysis}

The starting point is the standard description in terms of counter propagating waves, which in turn has been derived from first principles in a number of places (sec [10,13] for references) and is accepted today as a safe ground for a consistent weakly nonlinear theory. The physical variables are of the form

$$
\boldsymbol{u}=\left[A^{+}(x, t) \mathrm{e}^{\mathrm{i} \omega t+\mathrm{i} k x}+A^{-}(x, t) \mathrm{e}^{\mathrm{i} \omega \ell-\mathrm{i} k x}+\text { c.c. }\right] \boldsymbol{u}_{0}+\cdots,
$$

where $\omega$ is half the forcing frequency and $k$ is the corresponding wavenumber. The (complex) wave amplitudes $A^{ \pm}$ arc small and depend slowly in both space and time, namely $\left|A_{x x}^{ \pm}\right| \ll\left|A_{x}^{ \pm}\right| \ll\left|A^{ \pm}\right| \ll 1,\left|A_{t}^{ \pm}\right| \ll\left|A^{ \pm}\right|$, and satisfy amplitude equations of the form

$$
A_{i}^{ \pm}= \pm v_{g} A_{x}^{ \pm}-\varepsilon \alpha_{1} A^{ \pm}+\mathrm{i}\left(\alpha_{2}\left|A^{ \pm}\right|^{2}+\alpha_{3}\left|A^{\mp}\right|^{2}\right) A^{ \pm}+\mu \alpha_{4} \bar{A}^{\mp},
$$

where

$$
0<\mu \ll 1 \text { and } 0<\varepsilon \ll 1
$$

denote the amplitude of the parametric forcing and the decay rate duc to dissipation, respectively. The coefficients $v_{g}, \alpha_{1}>0, \alpha_{2}, \alpha_{3}$, and $\alpha_{4}>0$ instead are all of order unity and account for transport at the group velocity, linear dissipation, nonlinear (self and counter) interaction, and parametric forcing, respectively. Detuning due to dissipation could give a term proportional to $i \varepsilon A^{ \pm}$, but this is eliminated redefining the wavenumber $k$. Note that dissipation is only accounted for in that term proportional to $\varepsilon \Lambda^{ \pm}$, the remaining terms being Hamiltonian. The system (9) is imvariant under the actions

$$
\begin{aligned}
& x \rightarrow-x, \quad A^{+} \leftrightarrow A^{-} ; \quad x \rightarrow x+c_{1} \quad \text { for all } c_{1} ; \\
& \Lambda^{ \pm} \rightarrow \mathrm{e}^{ \pm \mathrm{i} c_{2}} \Lambda^{ \pm} ; \quad \text { and } t \rightarrow t+c_{3} \text { for all } c_{3},
\end{aligned}
$$

which result from invariance of the original problem under space reflection and translations. Note that these four actions generate a symmetry group that is larger than the original $O(2)$ group, the additional spurious symmetries (one of the actions associated with $c_{1}$ and $c_{2}$, and the action associated with $c_{3}$ ) resulting from truncation.

For sufficiently small $\left|A_{-}^{ \pm}\right|$(namely, sufficiently close to threshold) we have $\left|A^{+}\right| \simeq\left|A^{-}\right|$. Thus $A^{ \pm}$can be

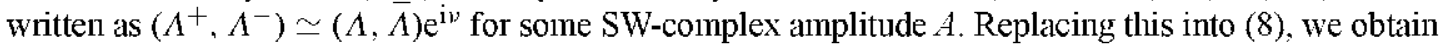

$$
\boldsymbol{u}=2 \cos (\omega t+\nu)\left[\Lambda(x, t) \mathrm{e}^{\mathrm{i} k x}+\text { c.c. } \mid \boldsymbol{u}_{0}+\cdots .\right.
$$

This is nothing but the SW mentioned above. In fact, the threshold for the appearance of nontrivial waves is calculated as the instability limit of the trivial state $\Lambda^{ \pm}=0$. Setting $\left(\Lambda^{+}, \bar{\Lambda}^{-}\right)=\left(\Lambda_{0}^{+}, \bar{\Lambda}_{0}^{-}\right) \mathrm{e}^{\lambda t+\mathrm{i} \kappa x}$ in (9) and linearizing, we obtain that the most unstable mode is such that $\left|A_{0}^{+}\right|=\left|A_{0}^{-}\right|$(namely, a SW). The dispersion relation is

$$
\lambda=-\alpha_{1} \varepsilon+\sqrt{\alpha_{4}^{2} \mu^{2}-v_{g}^{2} \kappa^{2}} \simeq \alpha_{4}\left(\mu-\mu_{c}\right)-\frac{v_{g}^{2} \kappa^{2}}{2 \alpha_{1} \varepsilon},
$$


as $\kappa \ll \mu$ and $\varepsilon \ll 1$, where

$$
\mu_{c} \simeq \frac{\alpha_{1} \varepsilon}{\alpha_{4}}
$$

is the instability threshold amplitude. This gives the marginal instability curve (plotted on the right in Fig. 1) $\mu \simeq \sqrt{\alpha_{1}^{2} \varepsilon^{2}+v_{g}^{2} \kappa^{2}} / \alpha_{4} \simeq \mu_{c}+v_{g}^{2} \kappa^{2} /\left(2 \alpha_{1} \alpha_{4} \varepsilon\right)$. The bifurcated solutions are spatially uniform sWs of the form

$$
\Lambda^{ \pm}=R \mathrm{e}^{\mathrm{i}( \pm \kappa x+v)}
$$

where $R$ and $v$ are given by $\alpha_{1} \varepsilon=\alpha_{4} \mu \cos 2 v$ and

$$
\alpha_{1}^{2} \varepsilon^{2}+\left[v_{g} \kappa+\left(\alpha_{2}+\alpha_{3}\right) R^{2}\right]^{2}=\alpha_{4}^{2} \mu^{2},
$$

as obtained replacing (15) into (9) and climinating the phase $v$ in the resulting complex equation. Using this and (14) we see that as $\kappa=0$ and $R \rightarrow 0$,

$$
\alpha_{4} \varepsilon\left(\mu-\mu_{c:}\right) \simeq \frac{\left(\alpha_{2}+\alpha_{3}\right)^{2} R^{4}}{2 \alpha_{1}} .
$$

This and (13) suggest the following SW description near threshold

$$
A_{t}=\frac{\beta_{1}}{\varepsilon} A_{x x}+\beta_{0}\left(\mu-\mu_{c}\right) A-\frac{\beta_{2}}{\varepsilon}|A|^{4} A,
$$

where the SW-complex amplitude $A$ is as defined in (12) and

$$
\beta_{0}=\alpha_{4}, \quad \beta_{1}=\frac{v_{g}^{2}}{2 \alpha_{1}}, \quad \beta_{2}=\frac{\left(\alpha_{2}+\alpha_{3}\right)^{2}}{2 \alpha_{1}} .
$$

Note that since $\alpha_{1}>0$ and $\alpha_{4}>0$, these coefficients are all positive.

If $\kappa \neq 0$ a term $v_{g}\left(\alpha_{2}+\alpha_{3}\right) \kappa R^{2} / \alpha_{1}$ must be added in the right hand side of (17), which in turn yiclds a new term in the right hand side of (18), namely

$$
\mathrm{i} v_{g} \frac{\alpha_{2}+\alpha_{3}}{\alpha_{1} \varepsilon}|\Lambda|^{2} \Lambda_{x}
$$

which is comparable to the remaining terms in the limit (5) and has been always ignored in previous analyses. For consistency, a term proportional to $\left(|\Lambda|^{2}\right)_{x} \Lambda$ must also be added that has been also ignored. Thus (18) must be replaced by (6), where $\beta_{3}$ cannot be calculated from this simple analysis based on spatially uniform SWs, but

$$
\beta_{4}=-v_{g} \frac{\alpha_{2}+\alpha_{3}}{\alpha_{1}}
$$

Eq. (18) is not expected to apply for sufficiently small $\left|\mu-\mu_{c}\right|$ due to the absence of cubic terms. These appear because of higher order effects of dissipation and parametric forcing. The amplitude equations (9) must be replaced by

$$
\begin{aligned}
A_{t}^{ \pm}= & \pm\left(\gamma_{g}+\mathrm{i} \gamma_{1} \varepsilon\right) A_{x}^{ \pm}-\varepsilon \alpha_{1} A^{ \pm}+\left\lfloor\left(\mathrm{i} \alpha_{2}-\varepsilon \gamma_{2}\right)\left|A^{ \pm}\right|^{2}+\left(\mathrm{i} \alpha_{3}-\varepsilon \gamma_{3}\right)\left|A^{\mp}\right|^{2}\right\rfloor A^{ \pm} \\
& +\mu\left[\alpha_{4} \bar{A}^{\mp} \mp \mathrm{i} \gamma_{4} \bar{A}_{x}^{\mp}+\gamma_{5}\left|A^{ \pm}\right|^{2} \bar{A}^{\mp}+\gamma_{6}\left|A^{\mp}\right|^{2} \bar{A}^{\mp}+\gamma_{7}\left(A^{ \pm}\right)^{2} A^{\mp}\right\rfloor,
\end{aligned}
$$

where the new coefficients $\gamma_{1}, \ldots, \gamma_{7}$ are real. In particular,

$$
\gamma_{1}=\frac{\partial \alpha_{1}}{\partial k}, \quad \gamma_{4}=\frac{\partial \alpha_{4}}{\partial k} .
$$


Those linear terms proportional to $\mathrm{i} \varepsilon \Lambda_{x}^{ \pm}$and $\mathrm{i} \varepsilon \bar{\Lambda}_{x}^{\mp}$ account for the wavenumber dependence of $\alpha_{1}$ and $\alpha_{4}$ and are essential in the analysis below because they provide the wavenumber shift mentioned above. They were ignored both by Milner [12] and in all subsequent analyses, see [10]; this gap is perhaps a consequence of the fact that $\alpha_{1}$ and $\alpha_{4}$ are usually set to one by a (wavenumber-dependent!) rescaling. The new terms in (21) have been added taking into account:

i. That if $\mu$ is set to zero, then the problem is autonomous and thus is invariant under the action $\Lambda^{ \pm} \rightarrow \mathrm{e}^{\mathrm{i} c_{4}} \Lambda^{ \pm}$ for all $c_{4}$.

ii. That the equations must be invariant under the actions (11).

iii. The form of the forcing term, assumed monochromatic, proportional to $\mu \sin 2 \omega t$, which excludes an additional term proportional to $\mu\left(\bar{\Lambda}^{\mp}\right)^{2} \bar{\Lambda}^{ \pm}$that is also invariant under (11).

iv. That the appropriate scaling is such that $\mu \sim \varepsilon \ll 1,|\partial / \partial t| \ll \varepsilon,|\partial / \partial x| \ll \varepsilon,\left|A^{ \pm}\right|^{2} \ll \varepsilon$, sec Eqs. (42)-(43) below. This excludes higher order terms like, e.g., dispersion, which would give a term proportional to $i A_{x x}^{ \pm}$, and some terms that are quadratic in $\Lambda^{ \pm}$and linear in $\Lambda_{x}^{ \pm}$.

In principle, Eq. (21) should also include some (omitted) additional terms that are of the same order as those considered and are discussed now. Viscous effects and parametric forcing lead to (i) a detuning term proportional to

$$
\mathrm{i} \delta \Lambda^{ \pm}
$$

(where $\delta \ll \varepsilon$ may depend on $\varepsilon$ ), which is eliminated by a near-identity redefinition of the wavenumber $k$ in $(8)$; and (ii) some small corrections to $v_{g}, \alpha_{1}, \ldots$, and $\alpha_{5}$, which are eliminated by near-identity redefinition of these coefficients. (iii) An additional term proportional to i $\delta \bar{A}^{\mp}$ (with $\delta \ll \mu$ ) is climinated by a near-identity change of the phase of $A^{ \pm}$. And (iv) some additional terms proportional to

$$
\mathrm{i} \mu\left|\Lambda^{ \pm}\right|^{2} \bar{\Lambda}^{\mp}, \quad \mathrm{i} \mu\left|\Lambda^{\mp}\right|^{2} \bar{\Lambda}^{\mp}, \quad \mathrm{i} \mu\left(\Lambda^{ \pm}\right)^{2} \Lambda^{\mp}
$$

cannot be climinated in a simple way, but they do not contribute to the final SW-amplitude equation (as explained below) and are ignored from the outset for the sake of clarity.

Substitution of (15) into (21) and elimination of $v$ yields (cf (16))

$$
\left[\alpha_{1}+\gamma_{1} \kappa+\left(\gamma_{2}+\gamma_{3}\right) R^{2}\right]^{2} \varepsilon^{2}+\left[v_{g} k+\left(\alpha_{2}+\alpha_{3}\right) R^{2}\right]^{2}=\left[\alpha_{4}+\gamma_{4} \kappa+\left(\gamma_{5}+\gamma_{6}+\gamma_{7}\right) R^{2}\right]^{2} \mu^{2} .
$$

Note that those terms of the form (24) are eliminated introducing in the right hand side of (15) a factor $\mathrm{e}^{\mathrm{i} c R^{2}}$, for some appropriate constant $c$; this in turn gives new terms in (25) that are higher order, namely $O\left(\mu^{2} R^{6}\right)$, and can be neglected. Setting $R=0$ and $\kappa \sim \varepsilon^{2}$ in (25) we obtain the marginal instability curve (left curve in Fig. 1)

$$
\alpha_{4} \mu \simeq \alpha_{1} \varepsilon\left[1+\left(\frac{\gamma_{1}}{\alpha_{1}}-\frac{\gamma_{4}}{\alpha_{4}}\right) \kappa+\frac{v_{g}^{2} \kappa^{2}}{2 \alpha_{1}^{2} \varepsilon^{2}}\right] .
$$

The instability threshold is now (cf (14))

$$
\mu_{c}=\frac{\alpha_{1} \varepsilon}{\alpha_{4}}\left[1-\frac{\alpha_{1}^{2}}{2 v_{g}^{2}}\left(\frac{\gamma_{1}}{\alpha_{1}}-\frac{\gamma_{4}}{\alpha_{4}}\right)^{2} \varepsilon^{2}\right] \quad \text { at } \kappa_{c}=-\frac{\alpha_{1}^{2}}{v_{g}^{2}}\left(\frac{\gamma_{1}}{\alpha_{1}}-\frac{\gamma_{4}}{\alpha_{4}}\right) \varepsilon^{2} .
$$

Note that the correction on $\mu_{c}$ is negative and thus $\kappa_{c}$ : has a destabilizing cflect. Replacing $\kappa=\kappa_{c}$ into (25) and solving the resulting equation for $\varepsilon \ll 1$ and $R \ll 1$ we obtain, at leading order ( $\operatorname{cf}(17)$ ),

$$
\alpha_{4} \varepsilon\left(\mu-\mu_{c}\right)=\frac{\left(\alpha_{2}+\alpha_{3}\right)^{2} R^{4}+2 v_{g}\left(\alpha_{2}+\alpha_{3}\right) \kappa_{c} R^{2}}{2 \alpha_{1}}+\frac{\alpha_{4}\left(\gamma_{2}+\gamma_{3}\right)-\alpha_{1}\left(\gamma_{5}+\gamma_{6}+\gamma_{7}\right)}{\alpha_{4}} \varepsilon^{2} R^{2} .
$$


Using this, we can correct Eq. (18) to obtain Eq. (4), where $\beta_{0}, \beta_{1}, \beta_{2}, \beta_{4}$, and $\mu_{c}$ are given by (19), (20), and (27). The coefficient of the new cubic term is

$$
\beta_{5}=\beta_{50}+\beta_{51}
$$

where

$$
\beta_{50}=\frac{\alpha_{4}\left(\gamma_{2}+\gamma_{3}\right)-\alpha_{1}\left(\gamma_{5}+\gamma_{6}+\gamma_{7}\right)}{\alpha_{4}}, \quad \beta_{51}=\frac{v_{g}\left(\alpha_{2}+\alpha_{3}\right) \kappa_{c}}{\alpha_{1} \varepsilon^{2}}
$$

(with $\kappa_{6}$ as defined in (27)), as obtained taking into account (27) and (28). Note that $\beta_{51}$ is responsible for the $O(1)$-correction on $\beta_{5}$ mentioned above.

Eq. (4) can also be systematically derived from (21). This is done in the next section, where the still unknown coefficient $\beta_{3}$ will be also calculated and the expressions for the remaining coefficients will be checked.

\section{Asymptotic derivation of the SW-amplitude Eq. (4)}

Here, we derive the SW-amplitude Eq. (4) from the counterpropagating waves equations (21). In the distinguished limit (5), we use the following scalings for the slow space and time variables and the bifurcation parameter

$$
\xi=\varepsilon^{2} x, \quad \tau=\varepsilon^{3} t, \quad \alpha_{4}\left(\mu-\mu_{c}\right)=\varepsilon^{3} \Sigma .
$$

The forcing amplitude at threshold and the solution of (21) are expanded in powers of $\varepsilon$ as

$$
\alpha_{4} \mu_{c}=\varepsilon \alpha_{1}+\varepsilon^{2} s_{1}+\varepsilon^{3} s_{2}+\cdots, \quad \Lambda^{ \pm}=\varepsilon \Lambda_{0}^{ \pm}+\varepsilon^{2} \Lambda_{1}^{ \pm}+\varepsilon^{3} \Lambda_{2}^{ \pm}+\cdots .
$$

Replacing (31)-(32) into (21) and setting to \%ero the coefficient of cach power of $\varepsilon$, we obtain the following problems, at orders $\varepsilon^{2}, \varepsilon^{3}$, and $\varepsilon^{4}$,

$$
\begin{aligned}
& A_{0}^{ \pm}=\bar{A}_{0}^{\mp}, \\
& \alpha_{1}\left(A_{1}^{ \pm}-\bar{A}_{\mathrm{I}}^{\mp}\right)= \pm v_{g} A_{0 \xi}^{ \pm}+s_{1} A_{0}^{ \pm}+\mathrm{i}\left(\alpha_{2}+\alpha_{3}\right)\left|A_{0}^{ \pm}\right|^{2} A_{0}^{ \pm}, \\
& \alpha_{1}\left(A_{2}^{ \pm}-\bar{A}_{2}^{\mp}\right)=-A_{0 \tau}^{ \pm} \pm v_{g} A_{1 \xi}^{ \pm} \pm \mathrm{i}\left(\gamma_{1}-\frac{\alpha_{1} \gamma_{4}}{\alpha_{4}}\right) A_{0 \xi}^{ \pm}+\left(\Sigma+s_{2}\right) A_{0}^{+} \\
&-\left[\gamma_{2}+\gamma_{3}-\alpha_{1} \frac{\gamma_{5}+\gamma_{6}+\gamma_{7}}{\alpha_{4}}\right]\left|A_{0}^{ \pm}\right|^{2} A_{0}^{ \pm}+\mathrm{i} \alpha_{2}\left[2\left|A_{0}^{ \pm}\right|^{2} A_{1}^{ \pm}+\left(A_{0}^{ \pm}\right)^{2} \bar{A}_{1}^{ \pm}\right] \\
&+\mathrm{i} \alpha_{3}\left[\left|A_{0}^{ \pm}\right|^{2}\left(A_{1}^{ \pm}+\bar{A}_{1}^{\mp}\right)+\left(A_{0}^{ \pm}\right)^{2} A_{1}^{\mp}\right],
\end{aligned}
$$

where (33) and the solvability condition of (34), which is

$$
s_{1}=0,
$$

have been used in (35). The SW-amplitude equation is now obtained from the solvability condition of (35), which is obtained adding the Eq. (35) for $A_{2}^{+}-\bar{A}_{2}^{-}$to the complex conjugate of the equation for $A_{2}^{-}-\bar{A}_{2}^{+}$, dividing by 2 , and invoking (33) and (34). It follows that

$$
\begin{aligned}
0= & A_{0 \tau}^{+}-\frac{v_{g}}{2}\left(A_{1 \xi}^{+}-\bar{A}_{1 \xi}^{-}\right)-\mathrm{i} \frac{\gamma_{1} \alpha_{4}-\alpha_{1} \gamma_{4}}{\alpha_{4}} A_{0 \xi}^{+}-\left(2+s_{2}\right) A_{0}^{+}+\left[\gamma_{2}+\gamma_{3}-\alpha_{1} \frac{\gamma_{5}+\gamma_{6}+\gamma_{7}}{\alpha_{4}}\right]\left|A_{0}^{+}\right|^{2} A_{0}^{+} \\
& -\mathrm{i} \alpha_{2}\left|\Lambda_{0}^{+}\right|^{2}\left(\Lambda_{1}^{+}-\bar{\Lambda}_{1}^{-}\right)-\mathrm{i} \frac{\alpha_{2}-\alpha_{3}}{2}\left(\Lambda_{0}^{+}\right)^{2}\left(\bar{\Lambda}_{1}^{+}-\Lambda_{1}^{-}\right)
\end{aligned}
$$




$$
\begin{aligned}
= & A_{0 \tau}^{+}-\frac{v_{g}}{2 \alpha_{1}}\left[v_{g} A_{0 \xi}^{+}+\mathrm{i}\left(\alpha_{2}+\alpha_{3}\right)\left|A_{0}^{+}\right|^{2} A_{0}^{+}\right]_{\xi}-\mathrm{i} \frac{\gamma_{1} \alpha_{4}-\alpha_{1} \gamma_{4}}{\alpha_{4}} A_{0 \xi}^{+}-\left(\Sigma+s_{2}\right) A_{0}^{+} \\
& +\left[\gamma_{2}+\gamma_{3}-\alpha_{1} \frac{\gamma_{5}+\gamma_{6}+\gamma_{7}}{\alpha_{4}}\right]\left|A_{0}^{+}\right|^{2} A_{0}^{+}+\frac{\left(\alpha_{2}+\alpha_{3}\right)^{2}\left|A_{0}^{+}\right|^{4} A_{0}^{+}}{2 \alpha_{1}} \\
& -\frac{\mathrm{i}\left(\alpha_{2}+\alpha_{3}\right) v_{g}\left|A_{0}^{+}\right|^{2} A_{0 \xi}^{+}+\mathrm{i}\left(\alpha_{2}-\alpha_{3}\right) v_{g}\left(\left|A_{0}^{+}\right|^{2}\right) \xi A_{0}^{+}}{2 \alpha_{1}} .
\end{aligned}
$$

Note that that term proportional to i $\Lambda_{0 \xi}^{+}$provides the above mentioned shift in the wavenumber at threshold. In order to eliminate that term (and center the amplitude equation at threshold), we set

$$
A_{0}^{+}=A \exp \left[-\mathrm{i} \frac{\alpha_{1}^{2}}{v_{g}^{2}}\left(\frac{\gamma_{1}}{\alpha_{1}}-\frac{\gamma_{4}}{\alpha_{4}}\right) \xi\right]
$$

(which can be also written as $\Lambda_{0}^{+}=\Lambda \exp \left(i \kappa_{c} \xi / \varepsilon^{2}\right)$, with $\kappa_{c}$ given by (27)), to rewrite (37) as

$$
A_{T}=\beta_{1} A_{\xi \xi}+2 A-\beta_{2}|A|^{4} A-\mathrm{i} \beta_{3}\left(|A|^{2}\right)_{\xi} A-\mathrm{i} \beta_{4}|A|^{2} A_{\xi}-\beta \beta_{5}|A|^{2} A,
$$

provided that

$$
s_{2}=-\alpha_{1} \frac{\left(\gamma_{1} \alpha_{4}-\alpha_{1} \gamma_{4}\right)^{2}}{2 v_{g}^{2} \alpha_{4}^{2}},
$$

where $\beta_{1}, \beta_{2}, \beta_{4}$, and $\beta_{5}$ are as given in (19), (20), and (29), whilc

$$
\beta_{3}=-\frac{v_{g} \alpha_{2}}{\alpha_{1}} .
$$

Replacing $A$ by $\varepsilon^{-1} \Lambda$ into (39) (see Eqs. (32) and (38)) and taking into account the scalings (31), we obtain the amplitude Eq. (4), which was the main object of this section. As a byproduct, substituting (36) and (40) into (32), we obtain the value of the threshold amplitude $\mu_{c}$ anticipated above, in (27).

\subsection{Validity limits of the $S W$ amplitude equation}

For convenience, the SW equation has been derived in the distinguished limit (31), with $\left|\Lambda^{ \pm}\right| \sim \varepsilon$ (see (32)). This limit is distinguished because it leads to a SW equation in which all terms are of the same order. But the SW equation has a wider scope, namely it applies whenever

$$
\left|\mu-\mu_{c}\right| \ll \mu \sim \varepsilon,
$$

provided that

$$
\left|\frac{\partial}{\partial t}\right| \ll \varepsilon, \quad\left|\frac{\partial}{\partial x}\right| \ll \varepsilon, \quad\left|\Lambda^{ \pm}\right|^{2} \ll \varepsilon .
$$

These conditions result by inspection of the main ingredient in the analysis above, whose validity only requires that Eq. (21) reduce to equations of the form $\varepsilon \alpha_{1} \Lambda^{ \pm}-\mu \alpha_{4} \bar{\Lambda}^{\mp}=$ higher order terms, see (33)-(35). This in turn requires that the remaining terms in (21) be small compared to $\varepsilon\left|A^{ \pm}\right| \sim \mu\left|\bar{A}^{\mp}\right|$. Conditions (42)-(43) suggest to rescale (21) replacing $\mu \rightarrow \varepsilon \mu, t \rightarrow l / \varepsilon, x \rightarrow x / \varepsilon$, and $A^{ \pm} \rightarrow \sqrt{\varepsilon} A^{ \pm}$, which in turn leads to the following rescaled version of (4)

$$
\Lambda_{t}=\beta_{1} \Lambda_{x x}+\beta_{0}\left(\mu-\mu_{c}\right) \Lambda-\beta_{2}|\Lambda|^{4} \Lambda-\mathrm{i} \beta_{3}\left(|\Lambda|^{2}\right)_{x} \Lambda-\mathrm{i} \beta_{4}|\Lambda|^{2} \Lambda_{x}-\varepsilon \beta_{5}\left|\Lambda^{2}\right| \Lambda,
$$


in which we must still require that $\ldots \ll\left|\Lambda_{x x}\right| \ll\left|\Lambda_{x}\right| \ll|\Lambda| \ll 1$ and that $\left|\Lambda_{t}\right| \ll|\Lambda|$. Furthermore, it is readily seen that when repeating the derivation above with the new scaling all cocfficients in the SW amplitude equation exhibit bounded coefficients and thus the omitted terms (proportional to powers of $A, A_{x}, A_{x x}, \ldots$ ), are small compared to those displayed and can be safely neglected. Thus, the appearance of unbounded coefficients in (4) was only an artifact of scaling, which in Eq. (4) was chosen to coincide with that in the counterpropagating waves equations (9), to facilitate understanding the introductory analysis in Section 2.

Now we turn on the validity of the approximations (3) and (6) of (4). Cubic and quintic terms are comparable in (4) if $\beta_{2}|A|^{4} / \varepsilon \sim \varepsilon \beta_{5}|A|^{2} \sim \beta_{0}\left|\mu-\mu_{c}\right|$, which requires that

$$
\left|\mu-\mu_{c:}\right| \sim \frac{\beta_{5}^{2} \varepsilon^{3}}{\beta_{0} \beta_{2}} .
$$

For smaller values of $\left|\mu-\mu_{\mathcal{c}}\right|$ the quintic term can be neglected and the approximation (3) applies, while for larger values, the approximation (6) can be used. Note that (44) yields in practice extremely small values of $\left|\mu-\mu_{c}\right|$ if $\varepsilon$ is quite small.

\section{The Faraday system}

We consider a 2D, laterally unbounded, horizontal liquid layer of unperturbed depth $d^{*}$, which is vibrating vertically with an amplitude $\mu^{*}$ and a frequency $2 \omega^{*}$. For nondimensionalization of the Navier Stokes equations and boundary conditions, we use the characteristic time $\omega^{*-1}$ and the characteristic length $\ell^{*}$, defined as

$$
\omega^{* 2}=\frac{g}{\ell^{*}}+\frac{\sigma}{\rho \ell^{* 3}},
$$

where $g$ is the gravitational acceleration, $\sigma$ the surface tension, and $\rho$ is the density, all assumed constant. The resulting problem depends on four nondimensional parameters, namely

$$
d=\frac{d^{*}}{\ell^{*}}, \quad \mu=\frac{\mu^{*}}{\ell^{*}}, \quad S=\frac{\sigma}{\sigma+\rho g \ell^{* 2}}, \quad \text { and } \quad \varepsilon=\frac{v}{\omega^{*} \ell^{* 2}},
$$

which are the container's depth, the forcing amplitude, the gravity-capillary balance parameter, and a measure of viscous effects, respectively, where $v$ is the kinematic viscosity. Note that $S$ is such that $0 \leq S \leq 1$, and the cxtreme values $S=0$ and 1 correspond to the purely gravitational $(\sigma=0)$ and the purcly capillary $(g=0)$ limits, respectively. As above, see (10), we assume that both parametric forcing and dissipation be small, which require in particular that viscous effects be weak, namely that $\varepsilon$ be small. This in turn requires that both kinematic viscosity be small and the forcing frequency be not too large (for as $\omega^{*} \rightarrow \infty$, the denominator $\omega^{*} \ell^{* 2^{2}} \rightarrow 0$, see (45)); a complete quantitative analysis of the combined effects of viscosity and the forcing frequency in the Faraday instability threshold can be found in [14]. Also, we consider the limit of deep layer,

$$
d \gg 1
$$

which is the only one treated in the literature at this level. Note that the characteristic length $\ell^{*}$ has been defined in (45) such that $\omega(1)=1$, where $\omega(k)$ is given by the inviscid dispersion relation

$$
\omega=(1-S) k+S k^{3} \text {. }
$$

Thus the nondimensional frequency and wavenumber of the excited surface waves will be such that $\omega \simeq 1$ and $k \simeq 1$. If the nondimensional free surface elevation is written as (cf $(8)$ )

$$
f=\Lambda^{+}(x, t) \mathrm{e}^{\mathrm{i} \omega t+\mathrm{i} k x}+\Lambda^{-}(x, t) \mathrm{e}^{\mathrm{i} \omega t-\mathrm{i} k x}+\text { c.c. }+\cdots,
$$


then $A^{ \pm}$is given by the amplitude cquations (54) below, which reduce to (21) if the mean flow is ignored (as systematically done in the literature). Ignoring at the moment the mean flow, the following coefficients of (21) have been calculated repeatedly in various places ([5] and references given therein)

$$
\begin{array}{ll}
v_{g}=\frac{1+2 S}{2}, \quad \alpha_{1}=2, \quad \gamma_{1}=4, \quad \alpha_{4}=1, \quad \gamma_{4}=-1, \\
\alpha_{2}=\frac{3 S}{1-3 S}+\frac{8-3 S}{4}, \quad \alpha_{3}=-\frac{2}{1+3 S}-\frac{4+3 S}{2} .
\end{array}
$$

The nonlinear coefficients $\gamma_{2}, \gamma_{3}, \gamma_{5}, \gamma_{6}$, and $\gamma_{7}$ have been also calculated elsewhere ([10] and references therein), but we do not have enough confidence in these fairly involved calculations because the value of $\beta_{5}$ they provide is not correct, sec below. Thus, we cannot use Eq. (30) to obtain $\beta_{50}$, which is approximated selting $\varepsilon=10^{-5}$ in the counterpart of $\beta_{50}$ calculated in [9] for arbitrary $\varepsilon$. This is plotted with solid line in Fig. 2a, where it is compared with both its counterpart for the indicated finite values of $\varepsilon$ (plotted with dashed line, as calculated in [9]) and the value of $\beta_{5}$ obtained from current theories $[10,15]$. This latter comparison makes sense because the contribution $\beta_{51}$ has been ignored in $[10,15]$, and shows a $O(1)$ discrepancy; in particular, at $S=1$ (the only value of $S$ considered in [10]) the exact value of $\beta_{50}$ is 11.22 , while the value calculated in [10] is $\beta_{50}=12.0$. Note that the asymptotic value is quite good for $\varepsilon<0.005$, except in a vicinity of $S=1 / 3$, where $\beta_{50}$ diverges if $\varepsilon=0$ because of a well known 2:1 resonance (where the inviscid dispersion relation (48) is such that $\omega(2)=2 \omega(1)$ ), but takes large but finite values if $0<\varepsilon \ll 1$.

With (49) we can use (27) to calculate the wavenumber shift at threshold responsible for the effect described in Section 2 ,

$$
\kappa_{c}=\frac{-16 \varepsilon^{2}}{(1+2 S)^{2}} .
$$

This coincides with a part of the $O\left(\varepsilon^{2}\right)$ correction of the wavenumber at threshold calculated by Chen and Vinals [11Eq. (16)], which is

$$
k_{c}=\frac{(2 \varepsilon)^{3 / 2}}{1+2 S}-\frac{[6(1+2 S)+16] \varepsilon^{2}}{(1+2 S)^{2}}+\cdots .
$$

As anticipated just after Eq. (1), the difference between both expressions is a wavenumber shift due to viscous effects only, which has been eliminated in this paper by the near-identity wavenumber correction mentioned just after Eq. (23); this wavenumber correction produces a $O\left(\varepsilon^{3 / 2}\right)$ and a $O\left(\varepsilon^{2}\right)$ corrections to the threshold amplitude $\mu_{c}$. that have not been calculated above. The shift (50) instead is due to parametric forcing and produces the contribution $\beta_{51}$ to the cubic coefficient, which invoking (30) and (49) is given by

$$
\beta_{51}=\frac{4}{1+2 S}\left(\frac{2}{1+3 S}-\frac{3 S}{1-3 S}+\frac{9 S}{4}\right)
$$

This is plotted with solid line in Fig. 2b, where a comparison with its counterpart for indicated finite values of $\varepsilon$ (plotted with dashed line, as calculated in [9]) is also shown. Note that again the asymptotic value is fairly good for $\varepsilon<0.005$, except near $S=1 / 3$. For completeness, the whole coefficient $\beta_{5}=\beta_{50}+\beta_{51}$ is plotted with solid line in Fig. 2c, as calculated using Fig. 2a and b. This is also compared both with its counterpart for the indicated finite values of $\varepsilon$ taken from [11] and [9], which coincide between each other with great precision (a good indication that the calculations in both [11] and [9] are correct), and with the results from current asymptotic theories, which show a significant discrepancy; the discrepancy is due to both (a) a mistake in the asymptotic calculation of $\beta_{50}$ (sce Fig. 2a) and (b) the fact that the correction $\beta_{51}$ has been ignored. Note that $\beta_{5}$ is always positive, which means that the cubic term is always stabilizing in the Faraday system. 

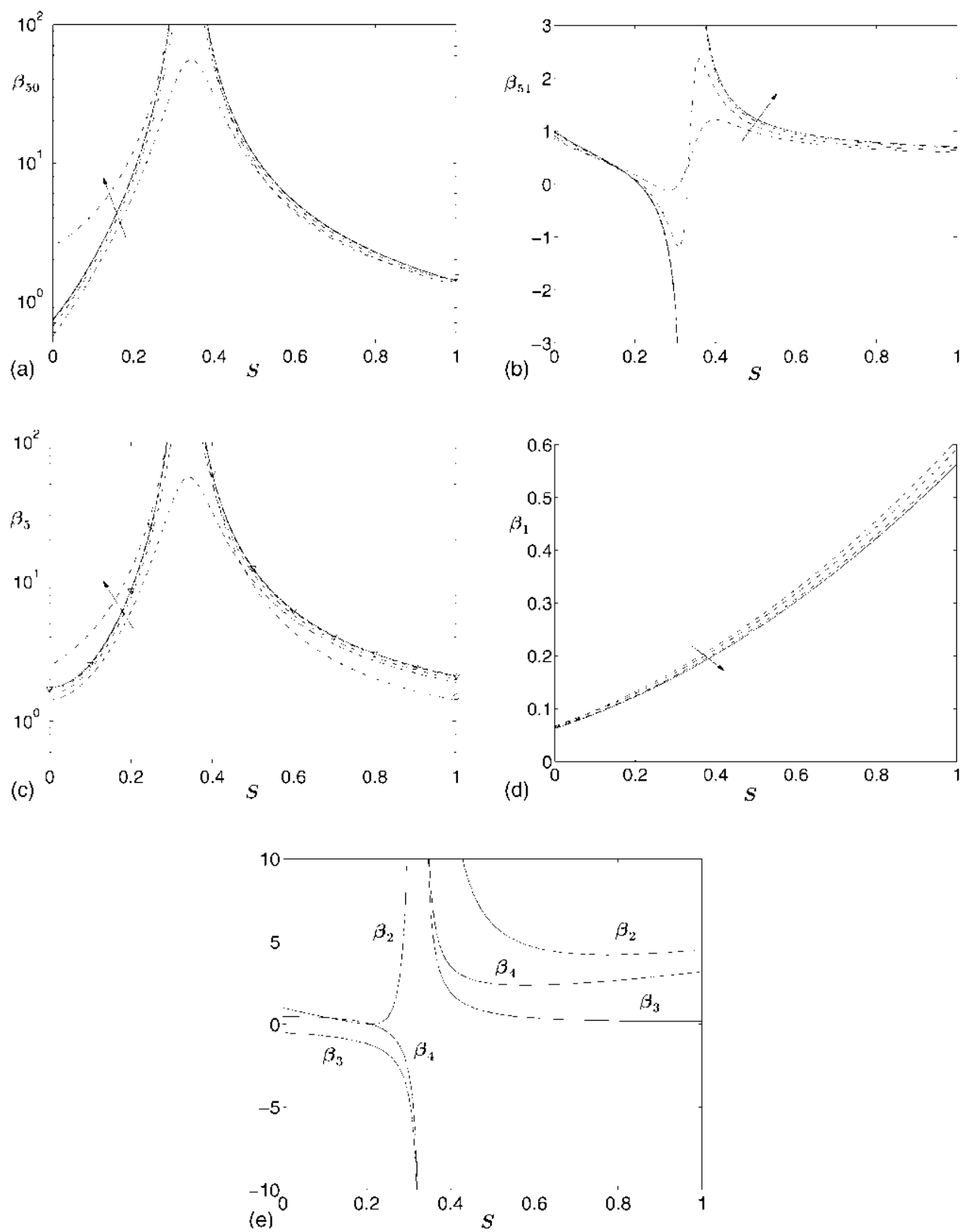

Fig. 2. The various coefficients of Eq. (39) for the Faraday system. (a) $\beta_{50}$, (b) $\beta_{51}$, (c) $\beta_{5}$, (d) $\beta_{1}$, and (e) $\beta_{2}, \beta_{3}$, and $\beta_{4}$. (一) as calculated in [9] for $\varepsilon=10^{5}$ (plot a) and as given by tqs. (52). (29), and (53) (plots b-e, respectively); (--) as calculated in [9] for $\varepsilon=1.25 \times 10^{2}$, $5 \times 10^{-3}$ and $5 \times 10^{-4}$ (the arrows indicate decreasing values of $\left.\varepsilon\right) ;(-\cdot--)$ as calculated in $\lceil 15]$ for $\varepsilon=0 ;(\nabla)$ as calculated in $[11]$ for $\varepsilon=5 \times 10^{-4} ;(0)$ as calculated ( for $\varepsilon=0$ ) in [10], where only the capillary limit was considered. 
Finally, using (19), (20), (41), and (49), we readily obtain the following expressions for the remaining coefficients in Eq. (39)

$$
\begin{aligned}
& \beta_{0}=1, \quad \beta_{1}=\frac{(1+2 S)^{2}}{16}, \quad \beta_{2}=\frac{1}{4}\left[\frac{3 S}{1-3 S}-\frac{2}{1+3 S}-\frac{9 S}{4}\right]^{2}, \\
& \beta_{3}=-\frac{1+2 S}{4}\left[\frac{3 S}{1-3 S}+\frac{8-3 S}{4}\right], \quad \beta_{4}=-\frac{1+2 S}{4}\left[\frac{3 S}{1-3 S}-\frac{2}{1+3 S}-\frac{9 S}{4}\right],
\end{aligned}
$$

which are plotted versus $S$ in Fig. $2 \mathrm{~d}$ and e. Note that $\beta_{1}$ compares well with its counterpart for finite $\varepsilon$ calculated in [9]; $\beta_{2}, \beta_{3}$, and $\beta_{4}$ instead do not have counterparts for finite $\varepsilon$, and (as $\beta_{5}$ does) diverge at the 2:1 resonance mentioned above. Also note that $\beta_{3}$ and $\beta_{4}$ change sign at $S=1 / 3$, while $\beta_{2}$ is always positive, as we already pointed out just after Eq. (19).

Unfortunately, the amplitude Eq. (39) only applies to the Faraday system for quite small $|\Sigma|$ (when in fact it reduces to the GL Eq. (70) below). For not so small $|\Sigma|$ we must add the effect of the viscous mean flow, which is considered next.

\section{1. Amplitude equations corrected by the mean flow}

At small viscosity, the Faraday system exhibits an oscillatory boundary layer attached to the free surface, which generates a viscous mean flow in the bulk. This mean flow in turn couples with the weakly nonlinear dynamics of the primary surface waves. This effect yields new terms in the amplitude equations (21), which to the approximation relevant here are

$$
\begin{aligned}
\Lambda_{t}^{ \pm}= & \pm\left(v_{g}+\mathrm{i} \gamma_{1} \varepsilon\right) \Lambda_{x}^{ \pm}-\varepsilon \alpha_{1} \Lambda^{ \pm}+\left[\left(\mathbf{i} \alpha_{2}-\varepsilon \gamma_{2}\right)\left|A^{ \pm}\right|^{2}+\left(\mathrm{i} \alpha_{3}-\varepsilon \gamma_{3}\right)\left|\Lambda^{\mp}\right|^{2} \pm 2 \mathrm{i} \int_{-d}^{0} \mathrm{e}^{2 y_{y}} \psi_{y}^{m} \mathrm{~d} y\right] \Lambda^{ \pm} \\
& +\mu\left[\alpha_{4} \bar{A}^{\mp} \mp \mathrm{i} \gamma_{4} \bar{A}_{x}^{\mp}+\gamma_{5}\left|A^{ \pm}\right|^{2} \bar{A}^{ \pm}+\gamma_{6}\left|A^{\mp}\right|^{2} \bar{A}^{\mp}+\gamma_{7}\left(A^{ \pm}\right)^{2} A^{\mp}\right] .
\end{aligned}
$$

Here, $\psi^{\mathrm{m}}$ is the streamfunction of the mean flow, defined such that the horizontal velocity is $-\psi_{y}^{\mathrm{m}} . \psi^{\mathrm{m}}$ is given by

$$
\psi_{y y y}^{\mathrm{m}}=0
$$

in $-d<y<0$, with boundary conditions

$$
\begin{aligned}
& \psi_{x}^{\mathrm{m}}-f_{l}^{\mathrm{m}}=2\left(\left|A^{-}\right|^{2}-\left|A^{+}\right|^{2}\right)_{x}, \quad \psi_{y y}^{\mathrm{m}}=8\left(\left|A^{+}\right|^{2}-\left|A^{-}\right|^{2}\right), \quad(1-S) f_{x}^{\mathrm{m}}+\varepsilon \psi_{y y y}^{\mathrm{m}}=0 \quad \text { at } y=0, \\
& \psi^{\mathrm{m}}=\psi_{y}^{\mathrm{m}}=0, \quad \text { at } y=-d,
\end{aligned}
$$

where $f^{\mathrm{m}}$ is the free surface deflection produced by the mean flow.

These equations are obtained adding to their counterparts derived in $[5]$ the higher order terms already considered in (21). Also:

- Since $d \gg 1(\sec (47))$, the effect of the boundary layer attached to the bottom of the container (which generates a $O\left(\mathrm{e}^{-2 d}\right)$-mean flow velocity) has been neglected. In fact, at the moment we only neglect $O\left(\mathrm{e}^{-2 d}\right)$-terms (which requires in practice that, say, $d \geq 2$ ) but kecp algebraically small terms in $d^{-1}$.

- In the boundary condition (56c) we have neglected capillarity, which gives a new term, $\varepsilon^{4} S f_{\xi \xi \xi}^{\mathrm{m}}$. Note that this cannot be neglected if $1-S=0$ or, more generally, if $|1-S|=O\left(\varepsilon^{4}\right)$. Thus we are excluding the case of too small gravitational effects.

- We anticipate that the mean flow is quitc weak ( $\left|\psi^{\mathrm{m}}\right| \sim \varepsilon^{3}$ and $\left|f^{\mathrm{m}}\right| \sim \varepsilon^{2}$, according to Eqs. (31)-(33), (58)-(59), (62), and (63)) and thus neglect convective terms in (55) and higher order terms in the boundary conditions. Also, invoking (31) we neglect time derivatives in (55). 
Because of these simplifications, we can integrate (55)-(57). It follows that

$$
\psi^{\mathrm{m}}=4(y+d)^{2}\left(\left|\Lambda^{-}\right|^{2}-\left|\Lambda^{+}\right|^{2}\right)+\frac{(1-S)(y+d)^{2}(2 d-y) f_{x}^{\mathrm{m}}}{6 \varepsilon}
$$

where $f^{\mathrm{m}}$ is given by

$$
f_{t}^{\mathrm{m}}=\frac{(1-S) d^{3} f_{x x}^{\mathrm{m}}}{3 \varepsilon}+2\left(1+2 d^{2}\right)\left(\left|\Lambda^{-}\right|^{2}-\left|\Lambda^{+}\right|^{2}\right)_{x}
$$

This latter equation shows that the slow motion of the free surface elevation is due to the restoring effect of gravity and the forcing effect of the free surface waves. Replacing (58) into (54) we obtain

$$
\begin{aligned}
\Lambda_{t}^{ \pm}= & \pm\left(v_{g}+\mathrm{i} \gamma_{1} \varepsilon\right) \Lambda_{x}^{ \pm}-\varepsilon \alpha_{1} \Lambda^{ \pm}+\left[\left(\mathrm{i} \alpha_{2}-\varepsilon \gamma_{2}\right)\left|A^{ \pm}\right|^{2}+\left(\mathrm{i} \alpha_{3}-\varepsilon \gamma_{s}\right)\left|\Lambda^{\mp}\right|^{2}\right] \Lambda^{ \pm} \\
& +\mu\left[\alpha_{4} \bar{A}^{\mp} \mp \mathrm{i} \gamma_{4} \bar{A}_{x}^{\mp}+\gamma_{5}\left|A^{ \pm}\right|^{2} \bar{A}^{\mp}+\gamma_{6}\left|A^{\mp}\right|^{2} \bar{A}^{\mp}+\gamma_{7}\left(A^{ \pm}\right)^{2} A^{\mp}\right] \\
& \pm \mathrm{i}\left[\gamma_{8}\left(\left|\Lambda^{-}\right|^{2}-\left|A^{+}\right|^{2}\right)+\varepsilon^{-1} \gamma_{9} f_{x}^{\mathrm{m}}\right] \Lambda^{ \pm},
\end{aligned}
$$

where we have neglected $O\left(\mathrm{e}^{-2 d}\right)$-terms and

$$
\gamma_{8}=4(2 d-1), \quad \gamma_{9}=\frac{(1-S)\left(2 d^{2}+1\right)}{4} .
$$

Now we extend the analysis in Section 3 to (60), using (31)-(32) and

$$
f^{\mathrm{m}}=\varepsilon^{2} \phi_{0}+\cdots,
$$

where the order of magnitude of $f^{\mathrm{m}}$ is anticipated from (59) and the following expression

$$
\left|A^{+}\right|^{2}-\left|A^{-}\right|^{2}=\varepsilon^{3}\left[\bar{A}_{0}^{+}\left(A_{1}^{+}-\bar{A}_{1}^{-}\right)+\text {c.c. }\right]+\cdots,
$$

which results invoking (32)-(33). Substituting (31)-(32), (58), and (62) into (60), we obtain again (33) and (34), but the following term must be added to the right hand side of (35)

$$
\pm \mathrm{i}\left[\gamma_{8} \bar{A}_{0}^{+}\left(A_{1}^{+}-\bar{A}_{1}^{-}\right)+\text {c.c. }+\gamma_{9} \phi_{0 \xi}\right] A_{0}^{ \pm} .
$$

Consequently, the following term must be added to (37)

$$
\mathrm{i}\left[\frac{\gamma_{8} v_{g}}{\alpha_{1}}\left(\left|A_{0}^{+}\right|^{2}\right)_{\xi} A_{0}^{+}+\gamma_{9} \phi_{0 \xi}\right] A_{0}^{ \pm},
$$

and introducing

$$
\phi=-\gamma_{9} \phi_{0} .
$$

we obtain (cf (39))

$$
A_{\tau}=\beta_{1} A_{\xi \xi}+2 A-\beta_{2}|A|^{4} A-\mathrm{i} \beta_{3}^{\prime}\left(|A|^{2}\right)_{\xi} A-\mathrm{i} \beta_{4}|A|^{2} A \xi-\beta \beta_{5}|A|^{2} A-\mathrm{i} \phi \xi A,
$$

where $\beta_{1}, \beta_{2}, \beta_{3}$ and $\beta_{5}$ are independent of the mean llow and thus are again as plotted in Fig. $2 \mathrm{c}-\mathrm{c}$, but

$$
\beta_{\mathfrak{3}}^{\prime}=\beta_{3}-\frac{\gamma_{8} v_{g}}{\alpha_{1}} \equiv \beta_{3}-(1+2 S)(2 d-1)
$$



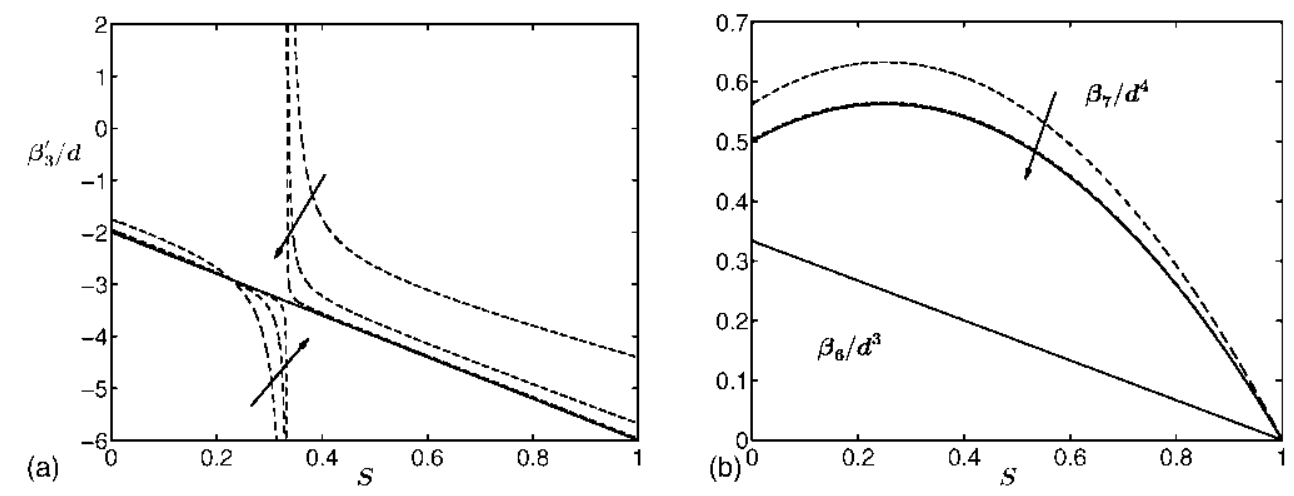

Fig. 3. The rescaled coefficients (a) $\beta_{3}^{\prime} / d$, and (b) $\beta_{6} / d^{3}$ (which is independent of $d$ ), and $\beta_{7} / d^{4}$ appearing in Eqs. (65) and (67), for (- - -) $d=2,10$ and 100 (1he arrows indicate increasing values of $d$ ), and $(-) d=\infty$, as calculated from $(66)$ and $(68)$.

shows a correction due to the viscous mean flow. Similarly, Eq. (59) can be written as

$$
\phi_{\tau}=\beta_{6} \phi_{\xi \xi}-\beta_{7}\left(|A|^{2}\right)_{\xi \xi},
$$

where

$$
\beta_{6}=\frac{(1-S) d^{3}}{3}, \quad \beta_{7}=2 \frac{\gamma_{9} v_{g}\left(1+2 d^{2}\right)}{\alpha_{1}} \equiv \frac{(1+2 S)(1-S)\left(1+2 d^{2}\right)^{2}}{8} .
$$

Because of volume conservation, the spatial mean value of $\phi$ must be zero, namely

$$
\langle\phi\rangle \equiv \lim _{L \rightarrow \infty} \frac{1}{L} \int_{0}^{L} \phi(\xi, \tau) \mathrm{d} \xi=0 .
$$

The (rescaled) coefficients $\beta_{3}^{\prime} / d, \beta_{6} / d^{3}$, and $\beta_{7} / d^{4}$ are plotted (vs. $S$, for the indicated values of $d$ ) in Fig. 3. Note that for finite $d, \beta_{3}^{\prime} / d$ diverges at the $2: 1$ resonance (at $S=1 / 3$ ) just because of the divergence of $\beta_{3}$ (see Fig. 2-e and Eq. (66)), and that $\beta_{7} / d^{4}$ approaches its asymptotic value quite rapidly as $d \rightarrow \infty$ (in fact, the curves for $d=100$ and $d=\infty$ are indistinguishable); $\beta_{6} / d^{3}$ instead is independent of $d$.

Summarizing, the dynamics of the surface waves are (coupled to the free surface elevation and) governed by Eqs. (65) and (67). Note that the coupling effect (namely, the last term in Eq. (65)) is produced by the viscous mean flow, and not by the mean free surface elevation itself. Namely, coupling comes from that term in (54) that depends on the mean flow horizontal velocity $\left(-\psi_{y}^{\mathrm{m}}\right)$, using Eq. (58) for $\psi^{\mathrm{m}}$. In addition, the viscous mean flow corrects the coefficient $\beta_{3}$ (see (66)).

As it happened in the absence of mean flow, we obtain the counterparts of Eqs. (3) and (6) for small and large $|\Sigma|$, respectively. In the former case, $|\partial / \partial \tau| \sim\left|\partial^{2} / \partial \xi^{2}\right| \sim|A|^{2} \sim|\phi| \sim|\Sigma| \ll 1$, and Eq. (65) becomes decoupled from (67) and simplifies to the following standard GL equation

$$
A_{\tau}=\beta_{1} A_{\xi \xi}+\Sigma A-\beta_{5}|A|^{2} A .
$$

Note that the mean flow plays no role in this limit. The limit of large $|\Sigma|$ leads to the scalings $|\partial / \partial \tau| \sim\left|\partial^{2} / \partial \xi^{2}\right| \sim$ $|A|^{4} \sim|\phi|^{1 / 2} \sim|\Sigma| \gg 1$. Thus all terms in (65) are comparable except the last two ones, which are smaller, and (65) is again decoupled from (67) and reduces to

$$
A_{\tau}=\beta_{1} A_{\xi \xi}+\Sigma A-\beta_{2}|A|^{4} A-\mathrm{i} \beta_{3}^{\prime}\left(|A|^{2}\right)_{\xi} A-\mathrm{i} \beta_{4}|A|^{2} A_{\xi} .
$$

This equation coincides with (6) except for the scaling and the value of the coefficient $\beta_{3}$, which is affected by the mean flow. 
Finally, we consider the limit of large $d$ (recall that $d$ was assumed only logarithmically large above). In this limit, $\beta_{3}^{\prime} \sim d, \beta_{6} \sim d^{3}$, and $\beta_{7} \sim d^{4}$ diverge as $d \rightarrow \infty$. Because of this, $\phi \sim d$ is also large, namely

$$
\phi=\frac{\beta_{7}}{\beta_{6}}\left(|A|^{2}-\left\langle|A|^{2}\right\rangle\right) \simeq \frac{6 v_{g} d}{\alpha_{1}}\left(|A|^{2}-\left\langle|A|^{2}\right\rangle\right),
$$

as obtained from (67) invoking (68)-(69). Also, those terms proportional to $\beta_{3}^{\prime}$ and $\phi_{\xi}$ dominate the remaining ones in the right hand side of (65), unless $\Sigma,|A|$, and $|\partial / \partial \xi|$ are small. The distinguished limit is

$$
T=L^{-2} \tau \sim L^{-1} \xi \equiv \zeta \sim \tilde{\Sigma} \equiv L^{2} \Sigma \sim B \equiv L A \sim 1 .
$$

Using this, (66), and (72), Eq. (65) simplifies to

$$
B_{T}=\beta_{1} B_{\zeta \zeta}+\tilde{\Sigma} B+\mathrm{i} \tilde{\beta}_{3} \frac{d}{L}\left(|B|^{2}\right)_{\zeta} B-\beta_{5}|B|^{2} B,
$$

where $\beta_{1}$ and $\beta_{5}$ are still as calculated above, but

$$
\tilde{\beta}_{3}=\frac{\gamma_{8} v_{g}}{\alpha_{1} d}-\frac{6 v_{g}}{\alpha_{1}}=\frac{1+2 S}{2} .
$$

Eq. (73) exactly coincides with (the limit for small viscosity of) its counterpart derived in [9], which applies as $d \gg 1$, without further restriction on $\varepsilon$. The coefficient $\tilde{\beta}_{3}$ is compared in Fig. 4 (as already done with $\beta_{1}$ in Fig. 2d) with its counterpart in [9] for the indicated (small but nonzero) values of $\varepsilon$. Note that the agreement is again quite good.

\subsection{Validity limits of the coupled $S W$ equations corrected by the mean flow}

The argument in Section 3.1 shows that the coupled system (54)-(57) applies whenever (42)-(43) hold and

$$
\left|\psi^{\mathrm{m}}\right| \ll \varepsilon
$$

This latter condition ensures in particular that inertia (resulting from the time derivative and convection) can be neglected in the momentum Eq. (55). And the same argument in Section 3.1 again shows that no further approximation applies if (44) holds. For smaller values of $\left|\mu-\mu_{c}\right|$, the approximation (70) can be used, while for larger values of $\left|\mu-\mu_{c}\right|$, the approximation (71) is safe.

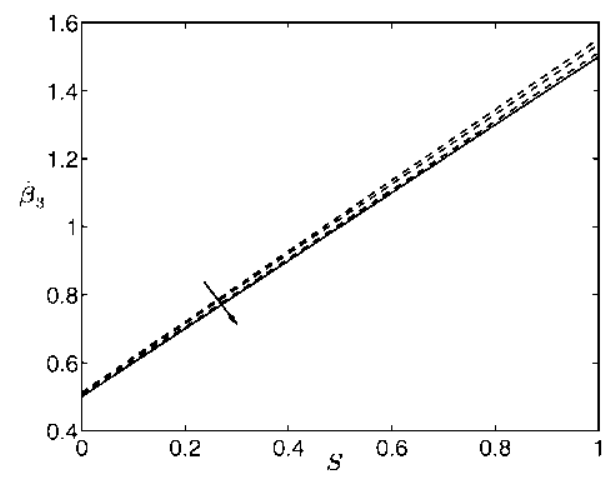

Fig. 4. The coefficient $\tilde{\beta}_{3}$ appearing in Fiq. (73): (-) as given by Eq. (74) and (-- ) as calculated by Mancebo and Vega [9] for $\delta=1.25 \times 10^{-2}$, $5 \times 10^{3}$, and $5 \times 10^{4}$ (the arrows indicate decreasing values of $\varepsilon$ ). 


\section{Concluding remarks}

We have obtained the quintic Eq. (4) that describes the dynamics of the SWs that appear near threshold in parametrically excited, nearly conservative, large aspect ratio systems that are invariant under $O(2)$. The coefficient of the quintic term $\beta_{2}>0$, as explained just after Eq. (19), which means that all solutions of (4) and (6) are bounded. The simultaneous presence of cubic and quintic terms in this equation does not require to be near a codimension-two point of the parameter space, but is a consequence of the smallness of dissipation (which of course can be seen as a codimension-two point of the parameter space of a fully dissipative system). We have shown that the cubic coefficient shows extreme dependence on wavenumber, which (a) implies that this coefficient must be calculated quite carefully in order to avoid wrong results, and (b) requires the presence of some cubic tems that involve the spatial derivative and have not been considered before. As explained in Section 1, these later terms prevent the existence of a Lyapunov function and break a spurious reflection symmetry, and thus have consequences in the dynamics. Eq. (4) only possesses the reflection symmetry (7), which implies in particular that all cocfficients, $\beta_{0}, \ldots, \beta_{5}$, are real. This means, in particular, that those terms proportional to $\beta_{1}, \beta_{2}$, and $\beta_{5}$ are dissipative, which could be surprising at first sight because these terms seem to come from Hamiltonian terms of the original counterpropagating waves Eqs. (21). But dissipation is implicitly present in all coefficients because that tcrm accounting for lincar damping in (21) (which must balance parametric forcing at leading order) plays a role in the derivation of all coefficients of (4).

Eq. (4) is invariant under the actions

$$
x \rightarrow-x, \quad A \rightarrow \bar{A} ; \quad x \rightarrow x+c_{1} \quad \text { for all } c_{1} ; \quad A \rightarrow A \mathrm{e}^{\mathrm{i} c_{2}} \quad \text { for all } c_{2} ; \quad t \rightarrow t+c_{3} \quad \text { for all } c_{3},
$$

which result from the symmetries (11). Thus no spurious symmetry results from truncation (associated with neglecting higher order terms in the derivation in Section 3), which is a good property to conjecture that (4) contains the whole dynamics of the original counterpropagating equations in the limit $\left|\mu-\mu_{\mathcal{C}}\right| \ll \varepsilon$, which is the natural scope of Eq. (4), as anticipated in Section 1 and explained in Section 3.1. In fact, Eq. (4) applies without further simplifications as $\left|\mu-\mu_{c}\right| \sim \varepsilon^{3}$; for smaller and larger values of $\left|\mu-\mu_{c}\right|$, this equation simplifies to the GL Eq. (3) and to the quintic Eq. (6), respectively.

The ideas above have becn applicd and extended to the Faraday sy stem. We have cncountered two gaps in previous calculations of the cubic coefficient, and have added the (systematically ignored in previous analy ses) effect of the mean flow, which is slaved to both (i) the surface waves and (ii) the free surface elevation it produces, see Eq. (58). Thus the mean flow has two effects, namely il (i) corrects the coefficient $\beta_{3}$ of Eq. (4) and (ii) couples (4) with a new amplitude equation giving the mean free surface elevation. The relevant amplitude equations, (65) and (67), have been derived from the pair of counterpropagating wave equations and the viscous mean flow equation, which in turn had been already obtained in [5]. The coefficients in the amplitude equations have been compared when possible (see Figs. 2b, d and 4) with their exact counterparts for finite $\varepsilon$ calculated in [9], with completely satisfactory results. Thus we are quite confident on the quantitative results above concerning the Faraday system.

As explained in Section 4.2, Eqs. (65) and (67) apply without further simplifications as $\left|\mu-\mu_{c}\right| \sim \varepsilon^{3}$ (namely, as (44) holds). For smaller values of $\left|\mu-\mu_{c}\right|$ the surface waves become decoupled from the mean flow and governed by the GL Eq. (70), which is also the relevant equation for fully dissipative Faraday waves in deep containers [9]. As $\left|\mu-\mu_{c \mid}\right| \gg \varepsilon^{3}$, the surface waves dy namics become decoupled from the mean flow (whose effect is only appreciated in the quantitative value of the coefficient $\beta_{3}^{\prime}$ ) and are governed by the quintic Eq. (71). This latter equation is the relevant one for comparison with experiments at quite low viscosity because for quite small $\varepsilon$ the restriction $\left|\mu-\mu_{c}\right|=O\left(\varepsilon^{3}\right)$ leads to extremely small values of $\left|\mu-\mu_{c}\right|$, which can be beyond experimental precision. In fact, at quite small viscosity, the experimentally observed primary bifurcated branch at threshold shows a quartic bchavior that suggests a purcly quintic nonlinearity [16] and not the quatratic behavior that should be expected from the cubic term. For instance, in the experiment by Douady, Fauve, and Thual [17] the liquid was water $(\sigma=72$ dyn $\mathrm{cm}^{-1}, v=0.01 \mathrm{~cm}^{2} \mathrm{~s}^{-1}, \rho=1 \mathrm{~g} \mathrm{~cm}^{-3}$ ) and the forcing frequency was $2 \omega^{*} \sim 40 \mathrm{~Hz}$. This gives invoking (45)- 
(46), (49), (53), and Fig. $2 \mathrm{c}, \varepsilon=2.2 \times 10^{-3}, \mu_{c}=2 \varepsilon, S=0.67$, and $\beta_{5}^{2} \varepsilon^{3} /\left(\beta_{0} \beta_{2}\right)=3.76 \times 10^{-6}$. Thus the relative precision for the effect of the cubic term to be appreciated is $\left|\mu-\mu_{c}\right| / \mu_{c} \sim 8.4 \times 10^{-4}$, which is seemingly beyond experimental precision. This is not always the case, of course. For instance, in the experiment by Westra, Binks, and van de Water [18], the liquid was a silicon oil $\left(\sigma=18 \mathrm{dyn} \mathrm{cm}^{-1}, \nu=0.036 \mathrm{~cm}^{2} \mathrm{~s}^{-1}, \rho=0.89 \mathrm{~g} \mathrm{~cm}^{-3}\right)$ and a forcing frequency was again $2 \omega^{*} \sim 40 \mathrm{~Hz}$; thus $\varepsilon=1.6 \times 10^{-2}, \mu_{c}=2 \varepsilon, S=0.54$, and $\beta_{5}^{2} \varepsilon^{3} /\left(\beta_{0} \beta_{2}\right)=5.0 \times 10^{-3}$, which give a much reasonable required precision, $\left|\mu-\mu_{c}\right| / \mu_{c} \sim 0.16$. This is consistent with the cubic behavior that was experimentally observed at threshold [18], according to the previous theory in $[15,11]$.

With these we can summarize the expected behavior of the Faraday sy stem near threshold for quite small viscosity. (i) As $\left|\mu-\mu_{c}\right| \ll \varepsilon^{3}$, the system will show the dynamically simple behavior governed by the Ginzburg-Landau cquation with real coefficients. (ii) As $\left|\mu-\mu_{c}\right| \sim \varepsilon^{3}$, nonpotential terms and coupling to the mean flow are expected to give complex dynamics resulting at least from oscillatory and Eckhaus instabilities. (iii) As $\varepsilon^{3} \ll\left|\mu-\mu_{c}\right| \ll \varepsilon$, coupling to the mean flow disappears but nonpotential terms remain and some (weaker) complexity is still expected. And (iv) as $\left|\mu-\mu_{i}\right| \sim \varepsilon$ or larger the standing wave description is not appropriate and the whole counterpropagating waves equations (coupled to highly nonlinear equations for the mean flow [5]) must be used. This whole picture could not be observed for (small but) finite values of $\varepsilon$. II, c.g., $\varepsilon=0.1$ only regime (i) (and perhaps a trace of regime (ii)) is to be expected.

The fact that the viscous mean flow directly affects the dynamics of the surface waves in the intermediate regime $\left|\mu-\mu_{c}\right| \sim \varepsilon^{3}$ but not as $\varepsilon^{3} \ll\left|\mu-\mu_{c}\right| \ll \varepsilon$ is surprising at first sight because for still larger values of $\left|\mu-\mu_{c}\right|$, namely as $\left|\mu-\mu_{c}\right| \sim \varepsilon$ or larger, the surface waves (which are no longer standing) become again coupled to the mean flow, as it comes out from the analysis of the counterpropagating waves in $[5,8]$. But the nature of this coupling is different from that encountered above, namely as $\left|\mu-\mu_{c}\right| \sim \varepsilon$, it is the mean flow itself and not the associated free surface elevation that affects the counterpropagating waves dynamics [5].

The mean flow and the new nonlinear terms do not affect the primary bifurcation from the flat state. Its effect must be appreciated in secondary bifurcations and in the resulting non trivial dynamics (e.g.. periodic attractors, which could not be present if both the mean flow and the new nonlinear terms were absent). It is promising that it is precisely in connection with these secondary bifurcations that experimental observations have not received a satisfactory theoretical explanation. This is true at large aspect ratio for one-dimensional waves in both anmular [17] and rectangular [19] containers, and for two-dimensional waves in 3D large aspect ratio containers [20]. For the sake of clarity, we have only considered the restricted case of one-dimensional waves, but the analysis above clcarly shows that (a) the cubic coefficient must show a similar dramatic dependence on wavenumber that has been not taken into account so far, and (b) both the new terms and the coupling to the mean flow must play an essential role also in 3D, and should help to give a convenient answer to the several theoretical problems that remain open today in connection with two-dimensional Faraday waves at large aspect ratio.

\section{Acknowledgement}

We are in debt with Dr. Peilong Chen for providing us with his calculations shown in Fig. 2c. This work was supported by the Spanish Dirección General de Enseñanza Superior under Grant BFM2001-2363 and by the National Aeronautics and Space Administration under Grant NNC04GA47G.

\section{References}

11] M. Faraday, (on the forms and states assumed by fluids in contact with vibrating elastie surfaces, Phil. Trans. R. Soc. 1,ondon 121 (1831) 319-340.

[2] J. Miles, 1). Henderson, Parametrically forced surfaee waves, Annu. Rev. Fluid Mech. 22 (1990) 143-165.

[3] M.C. Cross, P.C. IIohenberg, Patten formation oulside of equilibrium, Rev. Modern Phys. 65 (1993) 851-1112. 
[4] S. Fauve, Parametric instabilities, in: G. Martinez Mekler, T.H. Seligman (Eds.), Dynamics of Nonlinear and Disordered Systems, World Scientific, 1995 , pp. 67115 .

[5] J.M. Vega, F. Knobloch, C. Martel, Nearly inviseid Faraday waves in annular eontainers of moderately large aspect ratio, Physiea l) 154 (2001) 313-336.

[6] M.J. IIguera, J.M. Vega, E. Knobloch, Coupled amplitude-mean flow equations for nearly-inviscid Faraday waves in moderate aspect ratio conlainers, J. Nonlinear Sci. 12 (2002) 505-551.

[7] E. Martin, C. Martel, J.M. Vega, Drift instability of standing Faraday waves in an annular container, J. Fluid Mech. 467 (2002) 5779.

[8] V. Lapuerta, C. Martel, J.M. Vega, Weakly-dissipative Faraday waves in 2D large aspect ratio annuli, Physica D 173 (2002) 178203.

[9] F.J. Mancebo, J.M. Vega Viscous Faraday waves in 21) large aspeet ratio eontainers. I. Fluid Mech., Pre-print, 2004.

[10] P.I . Hansen, P. Alstrom, Perturbation theory of parametrically driven eapillary waves at low viscosity, I. Fluid Mech. 351 (1997) 301-344.

[11] P. Chen, J. Vinals, Amplitude equation and patlem selection in Faraday waves, Phys. Rev. E 60 (1999) 559-570.

[12] S.T. Milner, Square patterns and secondary instabilities in driven capillary waves, J. Fluid Mech. 225 (1991) 81100.

[13] C. Martel, E. Knobloch, J.M. Vega, Dynamics of counterpropagating waves in parametrically forced systems, Physica D 137 (2000) 94123.

[14] F.J. Mancebo, J.M. Vega, Faraday instability threshold in large aspect ratio containers, J. Fluid Mech. 467 (2002) 307-330.

[15] W. Thang, J. Viñals, Pattern formation in weakly damped parametric surfaee waves, J. Fluid Mcch. 336 (1997) 301-330.

[16] S. Fauve, private communication.

[17] S. Douady, S. Fauve, O. Thual, Oscillatory phase modulation of parametrically forced surface waves, Europhys. Lett. 10 (1989) 309-315.

[18] M.-T. Westra, D.J. Binks, W. van de Water, Patterns of Faraday waves, J. Fluid Mech. 496 (2003) 132.

[19] A.D.D. Craik, J. Armitage, Faraday excitation, hysteresis and wave instability in a narrow rectangular wave tank, Fluid Dyn. Res. 15 (1995) 129-143.

[20] A. Kudrolli, J.P. (iollub, Patterns and spatio-temporal chaos in parametrieally foreed surface waves: a systematic survey at large aspect ratio, Physica D 97 (1997) 133-154. 